\title{
The Fields of a Charged Particle in Hyperbolic Motion
}

\author{
Joel Franklin \\ David J. Griffiths \\ Department of Physics, Reed College \\ Portland, Oregon 97202
}

June 2, 2014

\begin{abstract}
A particle in hyperbolic motion produces electric fields that appear to terminate in mid-air, violating Gauss's law. The resolution to this paradox has been known for sixty years, but exactly why the naive approach fails is not so clear.
\end{abstract}

\section{Introduction}

In special relativity a particle of mass $m$ subject to a constant force $F$ undergoes "hyperbolic motion":

$$
z(t)=\sqrt{b^{2}+(c t)^{2}}
$$

where $b \equiv m c^{2} / F$. The particle flies in from infinity along (say) the $z$ axis, comes to rest at $z(0)=b$, and returns to infinity; its velocity approaches $\pm c$ asymptotically as $t \rightarrow \pm \infty$ (Figure 1 ).

Because information cannot travel faster than the speed of light, the region below the main diagonal $(z=-c t)$ is ignorant of the particle's existence - the particle is "over the horizon." For someone at the origin it first comes into view at $t=0$. If the particle is electrically charged, its fields are necessarily zero for all $z<0$, at time $t=0$. But the electric field for $z>0$ is not zero, and as we shall see the field lines appear to terminate in mid-air at the $x y$ plane 11 This would violate Gauss's law; it cannot be true. Our task is to locate the error and fix it ${ }^{2}$

\footnotetext{
${ }^{1}$ The entire $x y$ plane first "sees" the charge at time $t=0$. If this seems surprising, refer to Appendix A.

${ }^{2}$ The fields of a point charge in hyperbolic motion were first considered by M. Born, "Die Theorie des starren Elektrons in der Kinematik des Relativitätsprinzips," Ann. Physik 30, 1-56 (1909). For the early history of the problem see W. Pauli, Theory of Relativity (reprint by Dover, New York, 1981), Section 32( $\gamma)$. For a comprehensive history see E. Eriksen and $\varnothing$. Grøn, "Electrodynamics of Hyperbolically Accelerated Charges. I. The Electromagnetic
} 


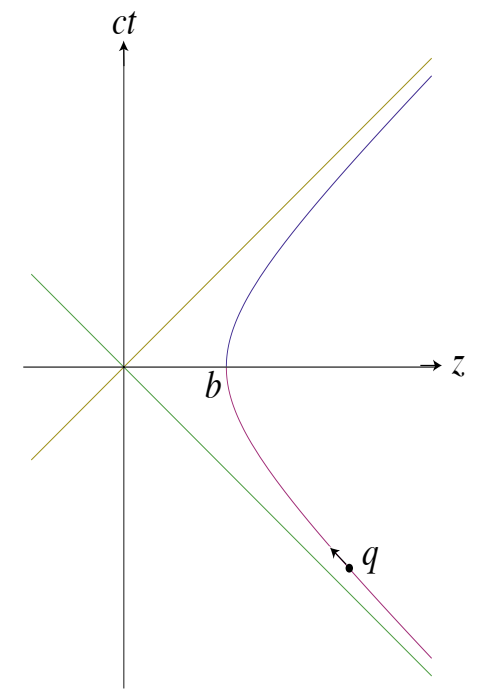

Figure 1: Hyperbolic motion.

In Section 2 we calculate the electric field of a charge $q$ in hyperbolic motion, at time $t=0$. A plot of the field lines shows that they do not go continuously to zero at the $x y$ plane. In Section 3 we explore the case of "truncated" hyperbolic motion (hyperbolic motion back to time $t=-t_{0}$, adjoined to constant velocity for earlier times). In this case the field lines make a sharp turn as they approach the $x y$ plane, and there is no violation of Gauss's law. In Section 4 we work out the potentials for a charge in hyperbolic motion, finding once again that we must adjoin "by hand" a term inspired by the truncated case. In Section 5 we ask how the naive calculations missed the extra term, and conclude with the puzzle unresolved. Appendices A and B supply some algebraic details, and Appendix $\mathrm{C}$ examines the radiation from a charge in hyperbolic motion; surprisingly, the "extra" terms do not contribute.

Field of a Charged Particle with Hyperbolic Motion," Ann. Phys. 286, 320-342 (2000). See also S. Lyle, Uniformly Accelerating Charged Particles: A Threat to the Equivalence Principle (Springer, Berlin, 2008). 


\section{Electric Field of a Charge in Hyperbolic Mo- tion}

We begin by calculating the electric field at the point $\mathbf{r}=(x, 0, z)$, with $z>0$. According to the standard formula $3^{3}$

$$
\mathbf{E}(\mathbf{r}, t)=\frac{q}{4 \pi \epsilon_{0}} \frac{\boldsymbol{r}}{(\boldsymbol{\imath} \cdot \mathbf{u})^{3}}\left[\left(c^{2}-v^{2}\right) \mathbf{u}+\boldsymbol{\imath} \times(\mathbf{u} \times \mathbf{a})\right],
$$

where

$$
\begin{gathered}
\mathbf{\imath}=x \hat{\mathbf{x}}+\left(z-\sqrt{b^{2}+\left(c t_{r}\right)^{2}}\right) \hat{\mathbf{z}}, \\
\mathbf{u}=c \hat{\mathbf{\imath}}-\mathbf{v}=\frac{1}{r}(c \boldsymbol{r}-r \mathbf{v}) \\
\mathbf{v}=\frac{c^{2} t_{r}}{\sqrt{b^{2}+\left(c t_{r}\right)^{2}}} \hat{\mathbf{z}}
\end{gathered}
$$

and

$$
\mathbf{a}=\frac{(b c)^{2}}{\left(\sqrt{b^{2}+\left(c t_{r}\right)^{2}}\right)^{3}} \hat{\mathbf{z}} .
$$

The retarded time, $t_{r}$, is defined in general by

$$
r=c\left(t-t_{r}\right),
$$

but for the moment we'll assume $t=0$ (so $t_{r}$ is negative). Then

$$
\left(c t_{r}\right)^{2}=x^{2}+\left(z-\sqrt{b^{2}+\left(c t_{r}\right)^{2}}\right)^{2}=x^{2}+z^{2}-2 z \sqrt{b^{2}+\left(c t_{r}\right)^{2}}+b^{2}+\left(c t_{r}\right)^{2},
$$

and hence

$$
c t_{r}=-\frac{1}{2 z} \sqrt{\left(x^{2}+z^{2}+b^{2}\right)^{2}-(2 z b)^{2}} .
$$

Putting all this together, and simplifying,

$$
\mathbf{E}(x, 0, z)=\frac{q b^{2}}{\pi \epsilon_{0}} \frac{\left(z^{2}-x^{2}-b^{2}\right) \hat{\mathbf{z}}+(2 x z) \hat{\mathbf{x}}}{\left(\sqrt{\left(z^{2}+x^{2}+b^{2}\right)^{2}-(2 z b)^{2}}\right)^{3}} .
$$

That is for $z>0$, of course; for $z<0$ the field is zero. In cylindrical coordinates $(s, \phi, z)$, then

$$
\mathbf{E}(s, \phi, z)=\frac{q b^{2}}{\pi \epsilon_{0}} \frac{\left(z^{2}-s^{2}-b^{2}\right) \hat{\mathbf{z}}+2 z \mathbf{s}}{\left(\sqrt{\left(z^{2}+s^{2}+b^{2}\right)^{2}-(2 z b)^{2}}\right)^{3}} \theta(z),
$$

where $\theta(z)$ is the step function ( 1 if $z>0$, otherwise 0 ). This field is plotted in Figure 2; the field lines are circles, centered on the $s$ axis and passing through the instantaneous position of the charge. 


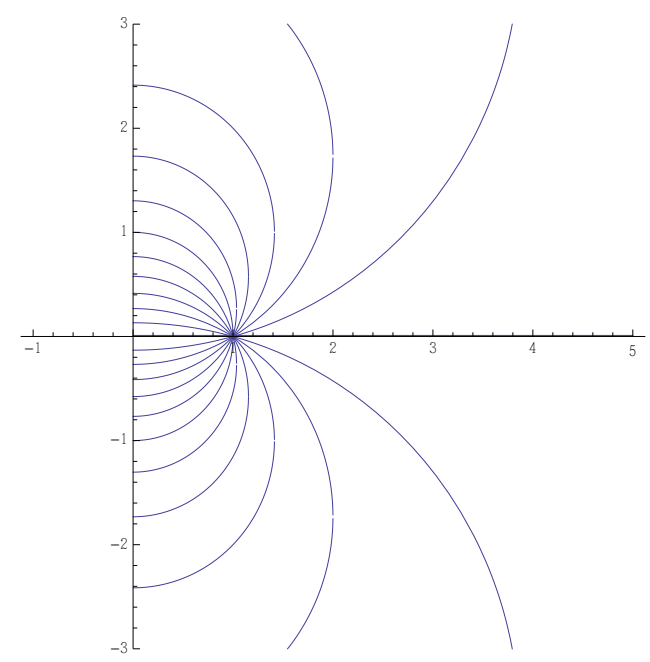

Figure 2: Field of a particle in hyperbolic motion, with $b=1$ (naive).

As required by Gauss's law, $\nabla \cdot \mathbf{E}=0$ for all $z>0$ (except at the point $s=$ $0, z=b$, where the charge is located). However, $\mathbf{E}$ is plainly not divergenceless at the $x y$ plane, where the field lines terminate in mid-air. Indeed, the field immediately to the right of the $z=0$ plane is

$$
\mathbf{E}\left(s, \phi, 0^{+}\right)=-\frac{q b^{2}}{\pi \epsilon_{0}} \frac{1}{\left(s^{2}+b^{2}\right)^{2}} \hat{\mathbf{z}},
$$

and the flux of $\mathbf{E}$ through a cylindrical Gaussian "pillbox" of radius $r$, centered at the origin and straddling the plane, with infinitesimal thickness, is

$$
\int \mathbf{E} \cdot d \mathbf{a}=-\frac{q b^{2}}{\pi \epsilon_{0}} \int_{0}^{r} \frac{1}{\left(s^{2}+b^{2}\right)^{2}} 2 \pi s d s=-\frac{q}{\epsilon_{0}}\left(\frac{r^{2}}{r^{2}+b^{2}}\right),
$$

even though the pillbox encloses no charge. Something is obviously amiss-we appear to have lost a crucial piece of the field at $z=0$.

\section{Truncated Hyperbolic Motion}

Suppose the acceleration does not extend all the way back to $t=-\infty$, but begins at time $t_{0}=-\alpha b / c$ (for some $\alpha>0$ ), when the particle was at

$$
z\left(t_{0}\right)=b \sqrt{1+\alpha^{2}},
$$

\footnotetext{
${ }^{3}$ See, for example, D. J. Griffiths, Introduction to Electrodynamics, 4th ed. (Pearson, Upper Saddle River, NJ, 2013), Eq. 10.72.

${ }^{4}$ This field was first obtained by G. A. Schott, Electromagnetic Radiation, Cambridge University Press, Cambridge, UK (1912), pp. 63-69.
} 
and its velocity was

$$
\mathbf{v}\left(t_{0}\right)=-\frac{\alpha c}{\sqrt{1+\alpha^{2}}} \hat{\mathbf{z}}
$$

prior to $t_{0}$ the velocity was constant. In other words, replace Eq. 1 with

$$
z(t)= \begin{cases}\frac{1}{\sqrt{1+\alpha^{2}}}(b-\alpha c t) & \left(t<t_{0}=-\alpha b / c\right) \\ \sqrt{b^{2}+(c t)^{2}} & \left(t \geq t_{0}\right) .\end{cases}
$$

At time $t=0$, for all points outside a sphere of radius $r=-c t_{0}=\alpha b$, centered at $z\left(t_{0}\right)$, the field is that of a charge moving at constant velocity - the "flattened" Heaviside field 5 radiating from the place $q$ would have reached, had it continued on its original flight plan $\left(b / \sqrt{1+\alpha^{2}}\right)$ :

$$
\mathbf{E}=\frac{q}{4 \pi \epsilon_{0}} \frac{1-(v / c)^{2}}{\left[1-(v / c)^{2} \sin ^{2} \theta\right]^{3 / 2}} \frac{\mathbf{R}}{R^{3}} .
$$

The left edge of the sphere is at $\left(\sqrt{1+\alpha^{2}}-\alpha\right) b$ (which is always positive, but goes to zero as $\alpha \rightarrow \infty$ ). Inside the sphere, where news of the acceleration has been received, the field is given by Eq. 11 (Figure 3). The field lines evidently join up in a thin layer at the surface of the sphere, representing the brief interval during which the motion switches from uniform to hyperbolic.

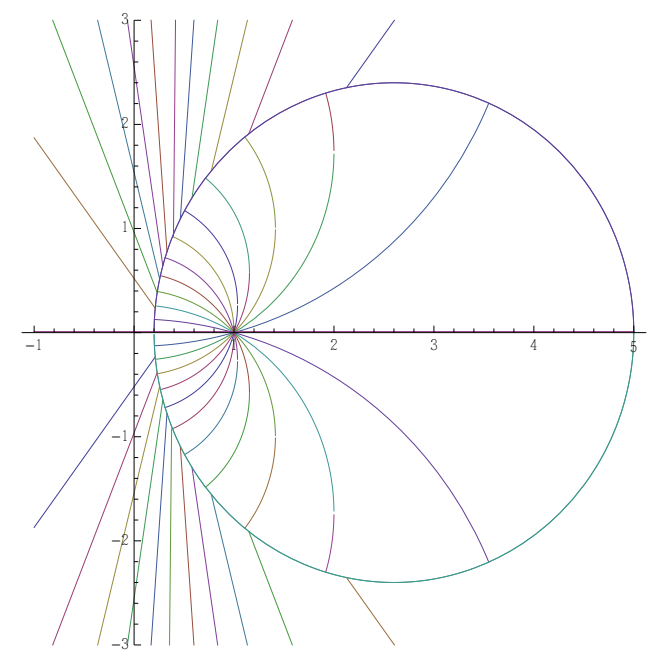

Figure 3: Field lines for truncated hyperbolic motion $(b=1, \alpha=12 / 5)$.

As alpha increases (that is, as $t_{0}$ recedes into the more distant past), the radius of the sphere increases, and its left surface flattens out against the $x y$

\footnotetext{
${ }^{5}$ E. M. Purcell and D. J. Morin, Electricity and Magnetism, 3rd ed. (Cambridge University Press, Cambridge, UK, 2013), Section 5.6.
} 


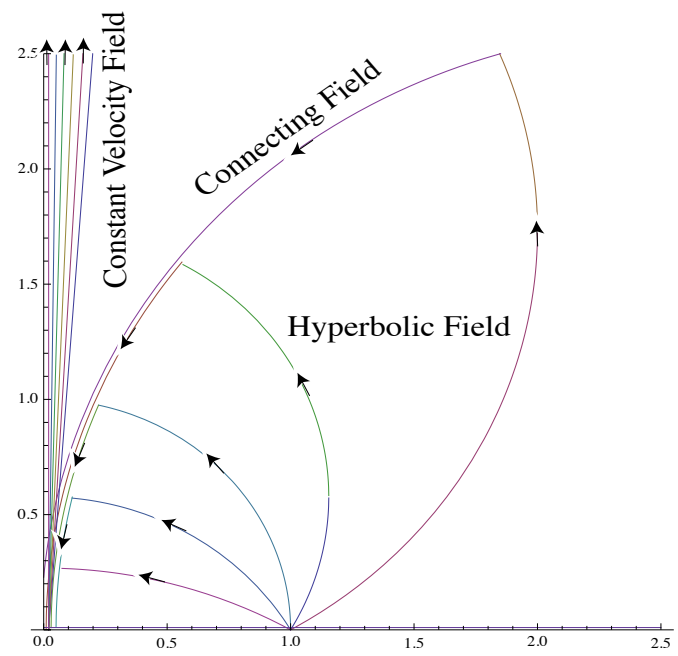

Figure 4: Truncated hyperbolic motion, for large $\alpha$, showing the "connecting" field.

plane. Meanwhile the "outside" field compresses into a disk perpendicular to the motion, and squeezes also onto the $x y$ plane. The complete field lines now execute a $90^{\circ}$ turn at $z=0$, as required to rescue Gauss's law. Indeed, for $\alpha \rightarrow \infty$ the constant velocity portion of the field approaches that of a point charge moving at speed $c^{6}$

$$
\mathbf{E}^{\prime}(s, \phi, z)=\frac{q}{2 \pi \epsilon_{0}} \frac{\mathbf{s}}{s^{2}} \delta(z) .
$$

Using the same Gaussian pillbox as before, this field yields

$$
\int \mathbf{E}^{\prime} \cdot d \mathbf{a}=\frac{q}{2 \pi \epsilon_{0}} \frac{r}{r^{2}}(2 \pi r) \int \delta(z) d z=\frac{q}{\epsilon_{0}} .
$$

This is appropriate, of course - had the particle continued at its original velocity (c) it would now be inside the box (at the origin).

Awkwardly, however, this is not what was needed to cancel the flux from the hyperbolic part of the field (Eq. 13). For that purpose the field on the $x y$ plane should have been

$$
\mathbf{E}(s, \phi, z)=\frac{q}{2 \pi \epsilon_{0}} \frac{\mathbf{s}}{s^{2}+b^{2}} \delta(z) .
$$

\footnotetext{
${ }^{6}$ J. M. Aguirregabiria, A. Hernández, and M. Rivas, " $\delta$-function converging sequences," Am. J. Phys. 70, 180-185 (2002), Eq. 50. The fields of a massless point charge are considered also in J. D. Jackson, Classical Electrodynamics, 3rd ed. (Wiley, New York, 1999), Prob. 11.18, W. Thirring Classical Mathematical Physics: Dynamical Systems and Field Theories (Springer-Verlag, New York, 1997), pp. 367-368, and M. V. Kozyulin and Z. K. Silagadze, "Light bending by a Coulomb field and the Aichelburg-Sexl ultraboost," Eur. J. Phys. 32, 1357-1365 (2011), Eq. 20.
} 
It must be that the "connecting" field in the spherical shell (the field produced during the transition from uniform to hyperbolic motion), which (in the limit) coincides with the $x y$ plane, and which we have ignored, accounts for the difference, as suggested in Figure 4. The net field in the $x y$ plane consists of two parts: the field $\mathbf{E}^{\prime}$ due to the portion of the motion at constant velocity, given (in the limit $\alpha \rightarrow \infty$ ) by Eq. 18, and the connecting field that joins it to the hyperbolic part. It is the sum of these fields that gives Eq. 20. The true field of a charge in hyperbolic motion is evidently 7

$$
\mathbf{E}(s, \phi, z)=\frac{q b^{2}}{\pi \epsilon_{0}} \frac{\left(z^{2}-s^{2}-b^{2}\right) \hat{\mathbf{z}}+2 z \mathbf{s}}{\left(\sqrt{\left(z^{2}+s^{2}+b^{2}\right)^{2}-(2 z b)^{2}}\right)^{3}} \theta(z)+\frac{q}{2 \pi \epsilon_{0}} \frac{\mathbf{s}}{s^{2}+b^{2}} \delta(z)
$$

and it does not look like Fig. 2, but rather Fig. 5 .

As a check, let's calculate the divergence of $\mathbf{E}$. Writing $\mathbf{E}=\mathbf{E}_{\theta} \theta(z)+\mathbf{E}_{\delta}$ (in an obvious notation), we have

$$
\nabla \cdot\left[\mathbf{E}_{\theta} \theta(z)\right]=\left(\nabla \cdot \mathbf{E}_{\theta}\right) \theta(z)+\mathbf{E}_{\theta} \cdot[\nabla(\theta)] .
$$

The first term gives $\rho / \epsilon_{0}$, for the point charge $q$ at $z=b$; as for the second term,

$$
\nabla \theta(z)=\frac{\partial \theta}{\partial z} \hat{\mathbf{z}}=\delta(z) \hat{\mathbf{z}}
$$

so

$$
\begin{aligned}
\nabla \cdot\left[\mathbf{E}_{\theta} \theta(z)\right] & =\frac{\rho}{\epsilon_{0}}+\frac{q b^{2}}{\pi \epsilon_{0}} \frac{\left(z^{2}-s^{2}-b^{2}\right)}{\left[\left(z^{2}+s^{2}+b^{2}\right)^{2}-(2 z b)^{2}\right]^{3 / 2}} \delta(z) \\
& =\frac{\rho}{\epsilon_{0}}-\frac{q b^{2}}{\pi \epsilon_{0}} \frac{1}{\left(s^{2}+b^{2}\right)^{2}} \delta(z) .
\end{aligned}
$$

Meanwhile

$$
\nabla \cdot \mathbf{E}_{\delta}=\frac{q}{2 \pi \epsilon_{0}} \nabla \cdot\left[\frac{\mathbf{s}}{s^{2}+b^{2}} \delta(z)\right]=\frac{q}{2 \pi \epsilon_{0}} \frac{1}{s} \frac{\partial}{\partial s}\left[\frac{s^{2}}{s^{2}+b^{2}} \delta(z)\right],
$$

so

$$
\nabla \cdot \mathbf{E}_{\delta}=\frac{q}{\pi \epsilon_{0}} \frac{b^{2}}{\left(s^{2}+b^{2}\right)^{2}} \delta(z) .
$$

This is just right to cancel the extra term in $\nabla \cdot\left[\mathbf{E}_{\theta} \theta(z)\right]$, and Gauss's law is sustained:

$$
\nabla \cdot \mathbf{E}=\frac{\rho}{\epsilon_{0}} .
$$

\footnotetext{
${ }^{7}$ The delta-function term was first obtained (using a somewhat different method) by H. Bondi and T. Gold, "The field of a uniformly accelerated charge, with special reference to the problem of gravitational acceleration," Proc. R. Soc. London Ser. A 229, 416-424 (1955). D. G. Boulware, "Radiation from a Uniformly Accelerated Charge," Ann. Phys. 124, 169-188 (1980) obtained it using the truncated hyperbolic model. The latter was also explored by W. Thirring A Course in Mathematical Physics: Classical Field Theory, 2nd ed. (Springer-Verlag, New York, 1992), p. 78. See also Lyle, ref. 2, Section 15.9.
} 


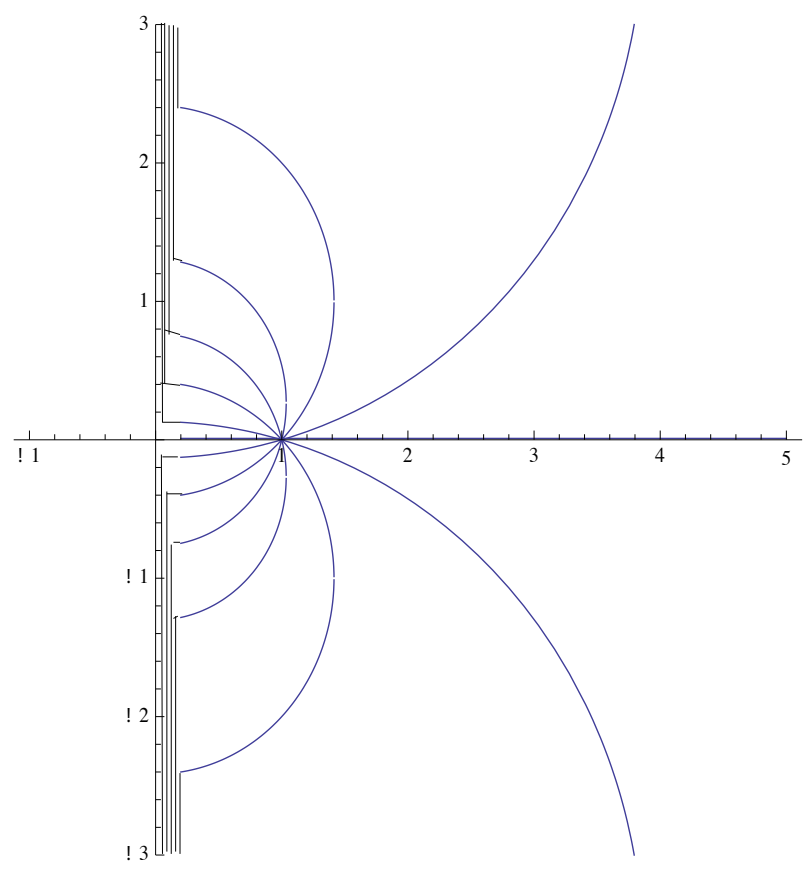

Figure 5: Field of a particle in hyperbolic motion (corrected).

\section{Potential Formulation}

\subsection{Liénard-Wiechert Potentials}

The truncated hyperbolic problem guided us to the "extra" (delta-function) term in Eq. 21, but it does not explain how we missed that term in the first place. Did it perhaps get lost in going from the potentials to the fields? Let's work out the Liénard-Wiechert potentials 8 and calculate the field more carefully:

$$
V(\mathbf{r}, t)=\frac{q}{4 \pi \epsilon_{0}} \frac{1}{[\boldsymbol{r}-(\boldsymbol{r} \cdot \mathbf{v}) / c]}, \quad \mathbf{A}(\mathbf{r}, t)=\frac{\mathbf{v}}{c^{2}} V(\mathbf{r}, t),
$$

where $\boldsymbol{\imath}$ and $\mathbf{v}$ are evaluated at the retarded time, $t_{r}$. For the point $\mathbf{r}=(\mathbf{s}, z)$,

$$
\begin{aligned}
T_{r}=\frac{-1}{2\left(T^{2}-z^{2}\right)}\left[T\left(s^{2}+z^{2}+b^{2}-T^{2}\right)\right. \\
\left.\quad-z \sqrt{4 b^{2}\left(T^{2}-z^{2}\right)+\left(s^{2}+z^{2}+b^{2}-T^{2}\right)^{2}}\right]
\end{aligned}
$$

\footnotetext{
${ }^{8}$ Reference 3, Eqs. 10.46 and 10.47 .
} 
$\left(T \equiv c t\right.$ and $\left.T_{r} \equiv c t_{r}\right){ }^{9}$ This is for $z>-T$; as we approach the horizon $(z \rightarrow-T)$, the retarded time goes to $-\infty$, and for $z<-T$ there is no solution with $T>T_{r}$.

The scalar potential is

$$
V=\frac{q}{4 \pi \epsilon_{0}} \frac{1}{\left(T^{2}-z^{2}\right)}\left[T-\frac{z\left(s^{2}+z^{2}+b^{2}-T^{2}\right)}{\sqrt{4 b^{2}\left(T^{2}-z^{2}\right)+\left(s^{2}+z^{2}+b^{2}-T^{2}\right)^{2}}}\right] \theta(T+z),
$$

and the vector potential is

$$
\mathbf{A}=\frac{q}{4 \pi \epsilon_{0} c} \frac{1}{\left(T^{2}-z^{2}\right)}\left[z-\frac{T\left(s^{2}+z^{2}+b^{2}-T^{2}\right)}{\sqrt{4 b^{2}\left(T^{2}-z^{2}\right)+\left(s^{2}+z^{2}+b^{2}-T^{2}\right)^{2}}}\right] \theta(T+z) \hat{\mathbf{z}} .
$$

The electric field is

$$
\begin{aligned}
\mathbf{E} & =-\nabla V-\frac{\partial \mathbf{A}}{\partial t} \\
& =\frac{q b^{2}}{\pi \epsilon_{0}}\left\{\frac{2 z \mathbf{s}-\left(s^{2}-z^{2}+b^{2}+T^{2}\right) \hat{\mathbf{z}}}{\left[4 b^{2}\left(T^{2}-z^{2}\right)+\left(s^{2}+z^{2}+b^{2}-T^{2}\right)^{2}\right]^{3 / 2}}\right\} \theta(T+z)
\end{aligned}
$$

(which reduces to Eq. 21-without the extra term-when $t=0$ ). Notice that the derivatives of the theta function contribute nothing (we use an overbar to denote the potentials shorn of their $\theta$ 's):

$$
\begin{aligned}
& -\delta(T+z)\left(\bar{V}+c \bar{A}_{z}\right)=-\frac{q}{4 \pi \epsilon_{0}} \delta(T+z) \frac{1}{\left(T^{2}-z^{2}\right)} \\
& \times\left[(T+z)-\frac{(T+z)\left(s^{2}+z^{2}+b^{2}-T^{2}\right)}{\sqrt{4 b^{2}\left(T^{2}-z^{2}\right)+\left(s^{2}+z^{2}+b^{2}-T^{2}\right)^{2}}}\right] \\
& =-\frac{q}{4 \pi \epsilon_{0}} \frac{\delta(T+z)}{(T-z)}\left[1-\frac{\left(s^{2}+b^{2}\right)}{\left(s^{2}+b^{2}\right)}\right]=0 .
\end{aligned}
$$

Evidently there is something wrong with the Liénard-Wiechert potentials themselves; they too are missing a critical term. To fix them, we play the same game as before: truncate the hyperbolic motion. We might as well go straight to the limit, with the truncation receding to $-\infty$; we need the potentials of a point charge moving at speed $c$. There are two candidates in the literature10 (which differ by a gauge transformation, though both satisfy the Lorenz condition, $\left.\partial V / \partial t=-c^{2}(\nabla \cdot \mathbf{A})\right)$

$$
V_{I}^{\prime}=0, \quad \mathbf{A}_{I}^{\prime}=-\frac{q}{2 \pi \epsilon_{0} c} \frac{\mathbf{s}}{s^{2}} \theta(z+T)
$$

\footnotetext{
${ }^{9}$ See Appendix B for details of these calculations. Equation 23 reduces to Eq. 9, of course, when $t=0$ and $s=x$.

${ }^{10}$ R. Jackiw, D. Kabat, and M. Ortiz, "Electromagnetic fields of a massless particle and the eikonal," Phys. Lett. B 277, 148-152 (1992).
} 


$$
V_{I I}^{\prime}=-\frac{q}{2 \pi \epsilon_{0}} \ln \left(\frac{s}{b}\right) \delta(z+T), \quad \mathbf{A}_{I I}^{\prime}=\frac{q}{2 \pi \epsilon_{0} c} \ln \left(\frac{s}{b}\right) \delta(z+T) \hat{\mathbf{z}}
$$

(in the second case $b$ could actually be any constant with the dimensions of length, but we might as well use a parameter that is already on the table).

We also need the "connecting" potentials; our experience with the fields (going from Eq. 18 to Eq. 20) suggests the following ansatz

$$
\begin{gathered}
V_{I}=0, \quad \mathbf{A}_{I}=-\frac{q}{2 \pi \epsilon_{0} c} \frac{\mathbf{s}}{\left(s^{2}+b^{2}\right)} \theta(z+T), \\
V_{I I}=-\frac{q}{4 \pi \epsilon_{0}} \ln \left(\frac{s^{2}+b^{2}}{b^{2}}\right) \delta(z+T), \quad \mathbf{A}_{I I}=\frac{q}{4 \pi \epsilon_{0} c} \ln \left(\frac{s^{2}+b^{2}}{b^{2}}\right) \delta(z+T) \hat{\mathbf{z}} .
\end{gathered}
$$

It is easy to check, in either case, that we recover the correct "extra" term in the field (Eq. 21). However, we prefer $V_{I I}$ and $\mathbf{A}_{I I}$, because they preserve the Lorenz gauge 11

The correct potentials for a point charge in hyperbolic motion are thus 12

$$
\begin{aligned}
V= & \frac{q}{4 \pi \epsilon_{0}}\left\{\frac{1}{\left(T^{2}-z^{2}\right)}\left[T-\frac{z\left(s^{2}+z^{2}+b^{2}-T^{2}\right)}{\sqrt{4 b^{2}\left(T^{2}-z^{2}\right)+\left(s^{2}+z^{2}+b^{2}-T^{2}\right)^{2}}}\right] \theta(T+z)\right. \\
& \left.-\ln \left(\frac{s^{2}+b^{2}}{b^{2}}\right) \delta(z+T)\right\}, \\
\mathbf{A}= & \frac{q}{4 \pi \epsilon_{0} c}\left\{\frac{1}{\left(T^{2}-z^{2}\right)}\left[z-\frac{T\left(s^{2}+z^{2}+b^{2}-T^{2}\right)}{\sqrt{4 b^{2}\left(T^{2}-z^{2}\right)+\left(s^{2}+z^{2}+b^{2}-T^{2}\right)^{2}}}\right] \theta(T+z)\right. \\
& \left.+\ln \left(\frac{s^{2}+b^{2}}{b^{2}}\right) \delta(z+T)\right\} \hat{\mathbf{z}} .
\end{aligned}
$$

How did the standard Liénard-Wiechert construction miss the extra (delta function) terms? Was it perhaps in the derivation of the Liénard-Wiechert potentials from the retarded potentials?

\subsection{Retarded Potentials}

Let's take a further step back, then, and examine the retarded potentia 13

$$
V(s, z)=\frac{1}{4 \pi \epsilon_{0}} \int \frac{\rho\left(\mathbf{r}^{\prime}, t_{r}\right)}{\imath} d^{3} \mathbf{r}^{\prime} .
$$

In this case

$$
\rho(\mathbf{r}, t)=q \delta^{3}\left(\mathbf{r}-\sqrt{b^{2}+(c t)^{2}} \hat{\mathbf{z}}\right)
$$

\footnotetext{
${ }^{11}$ Potentials 24 and 25 satisfy the Lorenz gauge condition, as does 31 , but 30 does not.

${ }^{12}$ The delta-function terms in the potentials were first obtained by T. Fulton and F. Rohrlich, "Classical radiation from a uniformly accelerated charge," Ann. Phys. 9, 499-517 (1960).

${ }^{13}$ Ref. 3, Eq. 10.19. Since all we're doing is searching for a missing term, we may as well concentrate on $V$, and set $t=0$.
} 
and we need $\rho\left(\mathbf{r}^{\prime}, t_{r}\right)$, where (for $t=0$ )

$$
-c t_{r}=\left|\mathbf{r}-\mathbf{r}^{\prime}\right|=\sqrt{\left(x-x^{\prime}\right)^{2}+\left(y-y^{\prime}\right)^{2}+\left(z-z^{\prime}\right)^{2}} .
$$

Thus

$$
\rho\left(\mathbf{r}^{\prime}, t_{r}\right)=q \delta^{3}\left(x^{\prime} \hat{\mathbf{x}}+y^{\prime} \hat{\mathbf{y}}+z^{\prime} \hat{\mathbf{z}}-\sqrt{b^{2}+\left(x-x^{\prime}\right)^{2}+\left(y-y^{\prime}\right)^{2}+\left(z-z^{\prime}\right)^{2}} \hat{\mathbf{z}}\right) .
$$

Because of the delta function, the denominator $\left(r=\left|\mathbf{r}-\mathbf{r}^{\prime}\right|\right)$ in Eq. 34 comes outside the integral - with $\mathbf{r}^{\prime}$, now, at the retarded point (where the argument of the delta-function vanishes). What remains is

$$
\begin{aligned}
Q & \equiv \int \rho\left(\mathbf{r}^{\prime}, t_{r}\right) d^{3} \mathbf{r}^{\prime} \\
& =q \int \delta\left(x^{\prime}\right) \delta\left(y^{\prime}\right) \delta\left(z^{\prime}-\sqrt{b^{2}+\left(x-x^{\prime}\right)^{2}+\left(y-y^{\prime}\right)^{2}+\left(z-z^{\prime}\right)^{2}}\right) d x^{\prime} d y^{\prime} d z^{\prime} \\
& =q \int \delta\left(z^{\prime}-\sqrt{b^{2}+s^{2}+\left(z-z^{\prime}\right)^{2}}\right) d z^{\prime}=q \int \delta\left(f\left(z^{\prime}\right)\right) d z^{\prime}
\end{aligned}
$$

where $s^{2}=x^{2}+y^{2}$, and

$$
f\left(z^{\prime}\right) \equiv z^{\prime}-\sqrt{b^{2}+s^{2}+\left(z-z^{\prime}\right)^{2}} .
$$
0 :

The argument of the delta function vanishes when $z^{\prime}=z_{0}$, given by $f\left(z_{0}\right)=$

$$
z_{0}=\sqrt{b^{2}+s^{2}+\left(z-z_{0}\right)^{2}}, \quad z_{0}^{2}=b^{2}+s^{2}+z^{2}-2 z z_{0}+z_{0}^{2},
$$

or

$$
z_{0}=\frac{1}{2 z}\left(s^{2}+z^{2}+b^{2}\right) .
$$

Note that $z_{0}$ is non-negative, so there is no solution when $z<0$. Now

$$
\frac{d f}{d z^{\prime}}=1+\frac{\left(z-z^{\prime}\right)}{\sqrt{b^{2}+s^{2}+\left(z-z^{\prime}\right)^{2}}}
$$

so

$$
f^{\prime}\left(z_{0}\right)=1+\frac{\left(z-z_{0}\right)}{z_{0}}=\frac{z}{z_{0}}=\frac{2 z^{2}}{s^{2}+z^{2}+b^{2}},
$$

and hence

$$
\delta\left(f\left(z^{\prime}\right)\right)=\frac{1}{\left|f^{\prime}\left(z_{0}\right)\right|} \delta\left(z^{\prime}-z_{0}\right)=\left(\frac{s^{2}+z^{2}+b^{2}}{2 z^{2}}\right) \delta\left(z^{\prime}-z_{0}\right) .
$$

Thus

$$
Q=q\left(\frac{s^{2}+z^{2}+b^{2}}{2 z^{2}}\right) \theta(z) .
$$

The retarded potential is

$$
V=\frac{1}{4 \pi \epsilon_{0}} \frac{Q}{r},
$$


and from Eq. 23 (with $t=0$ )

$$
r=-c t_{r}=-T_{r}=\frac{1}{2 z} \sqrt{\left(s^{2}+z^{2}+b^{2}\right)^{2}-(2 b z)^{2}},
$$

so

$$
V=\frac{q}{4 \pi \epsilon_{0}} \frac{\left(s^{2}+z^{2}+b^{2}\right)}{z \sqrt{\left(s^{2}+z^{2}+b^{2}\right)^{2}-(2 b z)^{2}}} \theta(z),
$$

and we recover Eq. 24 (for $t=0$ ). Still no sign of the extra term in Eq. 32; evidently the retarded potentials themselves are incorrect, in this case.

\section{$5 \quad$ What Went Wrong?}

Straightforward application of the standard formulas for the field (Eq. 2), the Liénard-Weichert potentials (Eq. 22), and the retarded potential (Eq. 34), yield incorrect results (inconsistent with Maxwell's equations) in the case of a charged particle in hyperbolic motion - they all miss an essential delta-function contribution. How did this happen? Bondi and Gold ${ }^{14}$ write,

"The failure of the method of retarded potentials to give the correct field is hardly surprising. The solution of the wave equation by retarded potentials is valid only if the contributions due to distant regions fall off sufficiently rapidly with distance."

Fulton and Rohrlich ${ }^{15}$ write,

"The Liénard/Wiechert potentials are not valid in the present case at $T+z=0$, because their derivation assumes that the source is not at infinity."

But where, exactly, do the standard derivations make these assumptions, and how can they be generalized to cover the hyperbolic case? ${ }^{16}$ Zangwil[17 offers a careful, step-by-step derivation of the retarded potentials; one of those steps must fail, but we have been unsuccessful in identifying the guilty party. And although it is easy to construct configurations for which the retarded potentials break down, we know of no other case for which the field formula (Eq. 2) fails.

Acknowledgement We thank Colin LaMont for introducing us to this problem 18

\footnotetext{
${ }^{14}$ Reference 7, quoted in Eriksen and Grøn, ref. 2.

${ }^{15}$ Reference 12, quoted in Eriksen and Grøn, ref. 2.

${ }^{16}$ Lyle (ref. 2, page 216) thinks it "likely" that the extra terms could in fact be obtained at the level of the Liénard-Wiechert potentials "if we were more careful about the step function," but he offers no justification for this conjecture.

${ }^{17}$ A. Zangwill, Modern Electrodynamics, Cambridge University Press, Cambridge (2013), Section 20.3.

${ }^{18}$ C. LaMont, "Relativistic Direct Interaction Electrodynamics: Theory and Computation," Reed College senior thesis, 2011.
} 


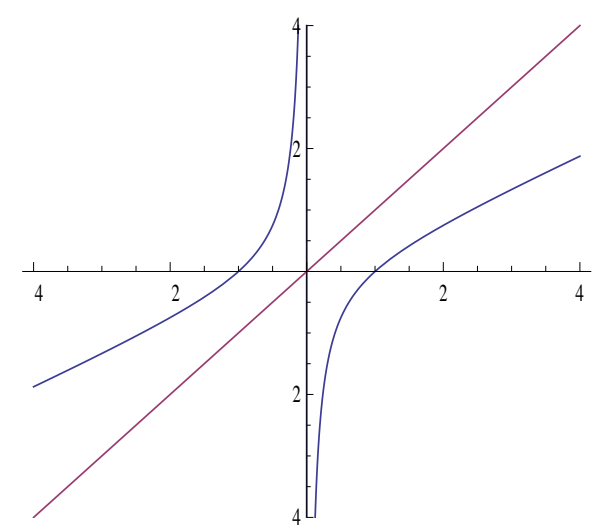

Figure 6: Graph of the retarded time, as a function of $t$, for points in the $x y$ plane.

\section{Appendices}

\section{A Retarded time for points on the $x y$ plane.}

The retarded time for a point in the $x y$ plane, at time $t$, is given by

$$
c\left(t-t_{r}\right)=\sqrt{x^{2}+y^{2}+z\left(t_{r}\right)^{2}}=\sqrt{x^{2}+y^{2}+b^{2}+c^{2} t_{r}^{2}},
$$

or

$$
c^{2} t^{2}-2 c^{2} t t_{r}+c^{2} t_{r}^{2}=x^{2}+y^{2}+b^{2}+c^{2} t_{r}^{2}
$$

so

$$
t_{r}=\frac{c^{2} t^{2}-x^{2}-y^{2}-b^{2}}{2 c^{2} t}=\frac{t^{2}-a^{2}}{2 t}, \quad \text { where } \quad a^{2} \equiv \frac{x^{2}+y^{2}+b^{2}}{c^{2}} .
$$

In Figure $6, t_{r}$ is plotted (as a function of $t$ ), for $a=1$. It is clear that $t_{r}<t$ for all positive $t$, but $t_{r}>t$ for all negative $t$. The latter is no good, of course, but we do get an acceptable solution for all $t \geq 0$. For $t=0$ the retarded time is (minus) infinity, regardless of the values of $x$ and $y$.

\section{B Potentials.}

The vector from the (retarded) position of the charge to the point $\mathbf{r}=(\mathbf{s}, z)$, is

$$
\boldsymbol{\imath}=\mathbf{s}+\left(z-\sqrt{b^{2}+T_{r}^{2}}\right) \hat{\mathbf{z}} .
$$


The retarded time is given by

$$
T-T_{r}=r=\sqrt{s^{2}+\left(z-\sqrt{b^{2}+T_{r}^{2}}\right)^{2}} .
$$

Squaring twice and solving the resulting quadratic yields Eq. 2319

Referring back to Eqs. 3 and 5, the denominator in Eq. 22 is

$$
d \equiv \boldsymbol{r}-\frac{\boldsymbol{\imath} \cdot \mathbf{v}}{c}=\left(T-T_{r}\right)-\left(z-\sqrt{b^{2}+T_{r}^{2}}\right) \frac{T_{r}}{\sqrt{b^{2}+T_{r}^{2}}}=T-\frac{z T_{r}}{\sqrt{b^{2}+T_{r}^{2}}} .
$$

It pays to use Eq. 49 to eliminate the radical:

$$
2 z \sqrt{b^{2}+T_{r}^{2}}=\left(s^{2}+z^{2}+b^{2}-T^{2}\right)+2 T T_{r},
$$

so

$$
d=T-\frac{2 z^{2} T_{r}}{\left(s^{2}+z^{2}+b^{2}-T^{2}\right)+2 T T_{r}} .
$$

Putting in Eq. 23, and simplifying,

$$
d=\frac{\left(T^{2}-z^{2}\right) \sqrt{\left(s^{2}+z^{2}+b^{2}-T^{2}\right)^{2}+4 b^{2}\left(T^{2}-z^{2}\right)}}{T \sqrt{\left(s^{2}+z^{2}+b^{2}-T^{2}\right)^{2}+4 b^{2}\left(T^{2}-z^{2}\right)}-z\left(s^{2}+z^{2}+b^{2}-T^{2}\right)},
$$

so

$$
V=\frac{q}{4 \pi \epsilon_{0}} \frac{1}{d}=\frac{q}{4 \pi \epsilon_{0}} \frac{1}{\left(T^{2}-z^{2}\right)}\left[T-\frac{z\left(s^{2}+z^{2}+b^{2}-T^{2}\right)}{\sqrt{4 b^{2}\left(T^{2}-z^{2}\right)+\left(s^{2}+z^{2}+b^{2}-T^{2}\right)^{2}}}\right]
$$

(Eq. 24).

Meanwhile, the vector potential (Eq. 22) is

$$
\begin{aligned}
\mathbf{A} & =\frac{\mathbf{v}}{c^{2}} V=\frac{T_{r}}{c \sqrt{b^{2}+T_{r}^{2}}} V \hat{\mathbf{z}}=\frac{2 z T_{r}}{c\left[\left(s^{2}+z^{2}+b^{2}-T^{2}\right)+2 T T_{r}\right]}\left(\frac{q}{4 \pi \epsilon_{0} d}\right) \hat{\mathbf{z}} \\
& =\left(\frac{q}{4 \pi \epsilon_{0} c}\right) \frac{2 z T_{r}}{T\left[\left(s^{2}+z^{2}+b^{2}-T^{2}\right)+2 T T_{r}\right]-2 z^{2} T_{r}} \hat{\mathbf{z}} \\
& =\frac{q}{4 \pi \epsilon_{0} c} \frac{1}{\left(T^{2}-z^{2}\right)}\left[z-T \frac{\left(s^{2}+z^{2}+b^{2}-T^{2}\right)}{\sqrt{4 b^{2}\left(T^{2}-z^{2}\right)+\left(s^{2}+z^{2}+b^{2}-T^{2}\right)^{2}}}\right] \hat{\mathbf{z}}
\end{aligned}
$$

(Eq. 25).

\footnotetext{
${ }^{19}$ The sign of the radical is enforced by the condition $T>T_{r}$.
} 


\section{Radiation}

From the potentials (Eqs. 32 and 33) we obtain the fields ${ }^{20}$

$$
\begin{aligned}
\mathbf{E}(s, \phi, z, t)= & \frac{q b^{2}}{\pi \epsilon_{0}}\left\{\frac{\left(z^{2}-s^{2}-b^{2}-T^{2}\right) \hat{\mathbf{z}}+2 z \mathbf{s}}{\left[\left(z^{2}+s^{2}+b^{2}-T^{2}\right)^{2}-4 b^{2}\left(z^{2}-T^{2}\right)\right]^{3 / 2}}\right\} \theta(z+T) \\
& +\frac{q}{2 \pi \epsilon_{0}} \frac{\mathbf{s}}{s^{2}+b^{2}} \delta(z+T) ; \\
\mathbf{B}(s, \phi, z, t)= & \left\{\frac{q b^{2}}{\pi \epsilon_{0} c} \frac{2 T s}{\left[\left(z^{2}+s^{2}+b^{2}-T^{2}\right)^{2}-4 b^{2}\left(z^{2}-T^{2}\right)\right]^{3 / 2}} \theta(z+T)\right. \\
& \left.-\frac{q}{2 \pi \epsilon_{0} c} \frac{s}{s^{2}+b^{2}} \delta(z+T)\right\} \hat{\boldsymbol{\phi}}=\frac{1}{c}(\hat{\boldsymbol{\imath}} \times \mathbf{E}) .
\end{aligned}
$$

To calculate the power radiated by the charge at a time $t_{r}$ (when it is located at the point $\left.z\left(t_{r}\right)=\sqrt{b^{2}-T_{r}^{2}}\right)$, we integrate the Poynting vector,

$$
\mathbf{S}=\frac{1}{\mu_{0}}(\mathbf{E} \times \mathbf{B}),
$$

over a sphere of radius $r=T-T_{r}$ centered at $z\left(t_{r}\right)$, and take the limit as $r \rightarrow \infty$, with $T_{r}=c t_{r}$ held constant. (That is, we track the energy as it flows outward at the speed of light; "radiation" is the portion that makes it "all the way to infinity.") We need the fields, then, at later and later times, as the sphere expands. Now, the delta-function term is confined to the plane $z=-T$, which recedes farther and farther to the left, as time goes on (Figure 7), and the expanding sphere never catches up. Curiously, then, the delta-function term does not contribute to the power radiated by the charge at any (finite) point on its trajectory. By the same token, the spherical surface is always in the region where $z+T>0$, so we can drop the theta functions.

Now,

$$
\mathbf{S}=\frac{1}{\mu_{0}}(\mathbf{E} \times \mathbf{B})=\frac{1}{\mu_{0} c}[\mathbf{E} \times(\hat{\boldsymbol{\imath}} \times \mathbf{E})]=\frac{1}{\mu_{0} c}\left[E^{2} \hat{\boldsymbol{\imath}}-(\hat{\boldsymbol{\imath}} \cdot \mathbf{E}) \mathbf{E}\right],
$$

and on the surface of the sphere $d \mathbf{a}=r^{2} \sin \theta d \theta d \phi \hat{\boldsymbol{\imath}}$ :

$$
\mathbf{S} \cdot d \mathbf{a}=\frac{1}{\mu_{0} c}\left[E^{2} \boldsymbol{r}^{2}-(\boldsymbol{r} \cdot \mathbf{E})^{2}\right] \sin \theta d \theta d \phi .
$$

The power radiated is 21

$$
P=\lim _{\boldsymbol{r} \rightarrow \infty}\left\{\frac{1}{\mu_{0} c} \int\left(1-\frac{\boldsymbol{r} \cdot \mathbf{v}}{\boldsymbol{r} c}\right)\left[E^{2} \boldsymbol{r}^{2}-(\boldsymbol{r} \cdot \mathbf{E})^{2}\right] \sin \theta d \theta d \phi\right\} .
$$

\footnotetext{
${ }^{20}$ Any reader with lingering doubts is invited to check that these fields satisfy all of Maxwell's equations. Note the critical role of the delta functions in Gauss's law and the Ampère-Maxwell law.

${ }^{21}$ The factor $(1-\boldsymbol{n} \cdot \mathbf{v} / \boldsymbol{r} c)$ accounts for the fact that the rate at which energy leaves a (moving) charge is not the same as the rate at which it (later) crosses a patch of area on the sphere. See ref. 3, page 485 .
} 
Using the relevant fields (Eq. 54) we find

$$
P=\lim _{r \rightarrow \infty}\left\{\left(\frac{r+T_{r}}{r}\right)^{2} \frac{c q^{2}}{6 \pi \epsilon_{0} b^{2}}\right\}=\frac{c q^{2}}{6 \pi \epsilon_{0} b^{2}} .
$$

Perhaps surprisingly, it is constant (independent of $T_{r}$, and hence the same for all points on the trajectory) ${ }^{22}$ It agrees with the Liénard formula ${ }^{23}$ (for collinear $\mathbf{v}$ and $\mathbf{a}$ ),

$$
P=\frac{q^{2}}{6 \pi \epsilon_{0} c^{3}} \gamma^{6} a^{2} .
$$

\footnotetext{
${ }^{22}$ The fact that a charged particle in hyperbolic motion radiates has interesting implications for the equivalence principle - in fact, it is this aspect of the problem that has attracted the attention of most of the authors cited here. Incidentally, the particle experiences no radiation reaction force- see R. Peierls, Surprises in Theoretical Physics (Princeton University Press, Princeton, NJ, 1979, Chapter 8.)

${ }^{23}$ Reference 3, Eq. 11.73.
} 
(a)

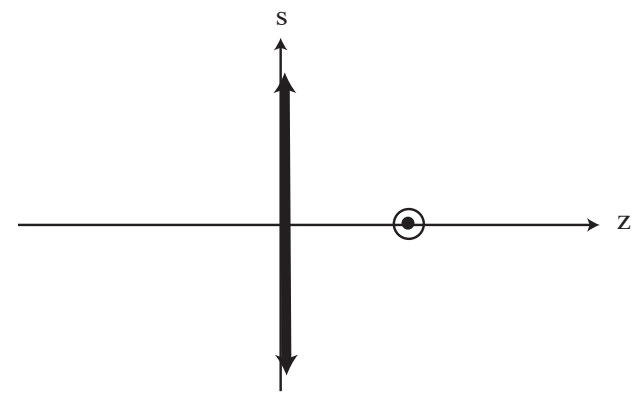

(b)

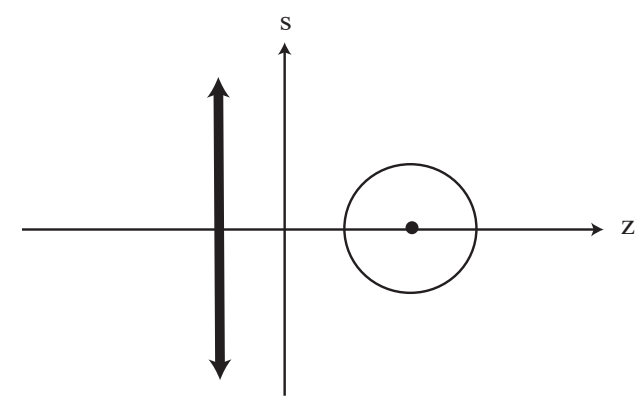

(c)

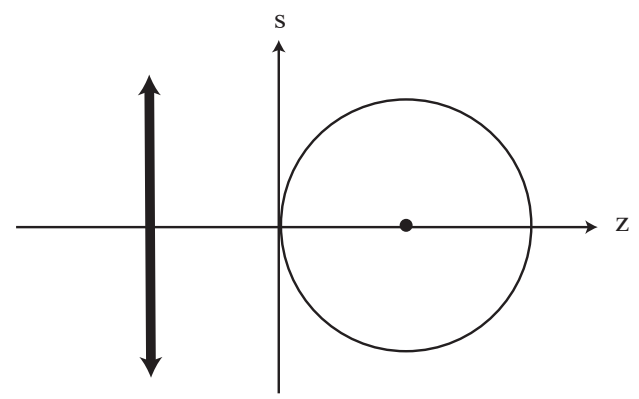

Figure 7: Radiation from the charge at time zero (for $b=1$ ), showing the spherical surface and the delta-fields at (a) $T=0$, (b) $T=1 / 2$, (c) $T=1$. 